\title{
A Novel OSA based ISM Band Channel Scanner Design for Identification and Deployment of White-Spaces
}

\author{
Nikhil P. Wyawahare ${ }^{1 *}$ and S. L. Haridas² \\ 'Department of Electronics Engineering, G. H. Raisoni College of Engineering, \\ Nagpur - 440016, Maharashtra, India; nikhil.wyawahare@raisoni.net \\ ${ }^{2}$ Department of Electronics and Telecommunication Engineering, G. H. Raisoni College of Engineering, \\ Nagpur-440016, Maharashtra, India; sanjay.haridas@raisoni.net
}

\begin{abstract}
Objectives: In this Paper a generic approach of ISM band channel scanner design module is proposed to identify available free white spaces to utilize under secondary communication network. Method/Analysis: The feature of opportunistic spectrum access (OSA) is to identify free channel to deployment purpose, the channel is compared on the basis of RSSI level individually amongst total available channel were in normally $80 \%$ channel are mostly vacant due to dominating nature of channel. The output of the OSA based ISM band channel brings a free channel identification and noise free path to continue communication in between other ISP. Findings: The result indicates that the available channels are having bunch of availability of total 14 no of channels in general. In which channel no 1-6 and 11 are comes under high dominating index ratio. The channel always forcing every new SSID/service provider to accommodate in between these three channels only; due to this a normal channel Co-channel conjunction/Interference occurs in crowed area. This may lead to call/request lost data packets in bulk! As a result users always share more data beyond a normal, causing more power consumption/ battery in cell phone/laptop. So for channel identification OSA technique is implemented which is actually determines dynamic spectrum access by comparing received power in $-\mathrm{dB}$ on $-90 \mathrm{~dB}$ to $-20 \mathrm{~dB}$ range were on results comparison above $-50 \mathrm{~dB}$ range signal is mostly having week strength/the origin of base channel is on far distance to other nearby channels. Once the low signal channel or non operational channel is detected it effectively deployed for secondary user network communication. Novelty/Improvements: In this novel design, portable OSA channel estimator-indicator is actively scanned in real time in live data fields; in future it helps to bi-pass the used channel to remove interference.
\end{abstract}

Keywords: White Space, ISM, OSA, RSSI, SSID

\section{Introduction}

\subsection{Mode of Design}

In wireless communication now a day's there are many wireless devices available on the market that broadcast in the $2.4 \mathrm{GHz}$ spectrum including Bluetooth, $802.11 \mathrm{a} / \mathrm{b}$ Ethernet Wi-Fi, Zigbee, wireless USB, cordless phones, wireless mice and keyboards and the microwave oven. Depending where people live in the world, the regulatory body has allocated a roughly some block for transmitting all manner of data starting at $2.400-2.483 \mathrm{GHz}$ frequency ${ }^{1}$. The problem occurred when it is getting a bit crowded in this band, especially for the people that live in a built up urban area. It is because; $2.4 \mathrm{GHz}$ band is over and

*Author for correspondence 
over again filled with the transmission of many different devices, all challenging for precious space and each one of it trying to make its signal heard above the noise. The reason of this problem was because the ISM band was an unlicensed band for many communications. Thus, a lot of wireless equipment maker have a propensity to make a creation that operate in this frequency to decrease the transmission cost $^{2}$. Spectrum analyzer is a gadget that able to displays the signal strength at each frequency step across a transmission band. It will be able to display the signal of ISM bands in many areas like rural, urban. The piece of equipment will also helps users to monitor quickly if there was any interfering signal present at the time. Besides of that, in term of safety matter, this device can be used to check if there is microwave leakage in your house. As a part of research $2.4 \mathrm{GHz}$ spectrum analyzer is design to commence a low cost portable and compact spectrum analyzer where this will be economically affordable, portable and easily handled by consumers in comparison to other similar product in the market. It also has high potential to be commercialized. Also broadband incursion is still very.
Low in developing countries such as India, especially in rural areas, because of the unaffordable cost of laying cable and fiber. Recently, wireless solutions based on Wi-Fi have been proposed as a cost efficient solution to bridge the last-mile problem.

\subsection{Motivation}

The increase in wireless data traffic is not only limited to the improved lifestyle and technological advancement but also in some worst case scenarios such as pre and post disaster emergency communications. In such situations, the present spectrum opportunities available are found to be inadequate to support a huge number of wireless data traffic that increases exponentially as people in terror communicating with their loved ones. In some cases like that happened on $26^{\text {th }}$ November 2008 Mumbai Terrorist Attack, the Walkie-Talkie used by the Mumbai Police department which works on half duplex principle was found to be inefficient for communication between the Police patrolling teams. There is a need for new spectrum opportunities. There are various portions of the already allocated spectrum remaining underutilized due to

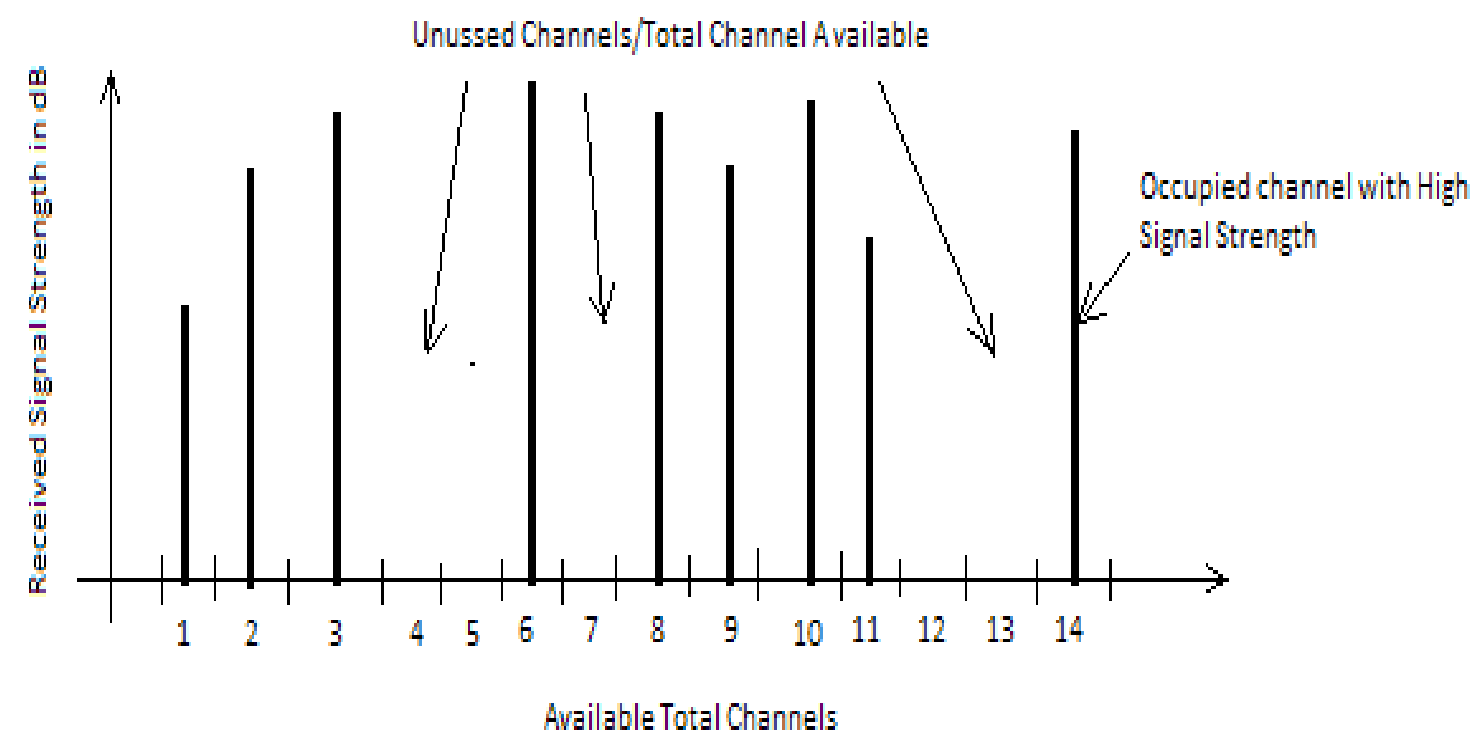

Figure 1. Available vaccant channel per total received channel in $\mathrm{dB}$. 
which regulators are now shifting their focus in increasing its use. Wi-Fi or Wireless Fidelity is a term that is used generically to refer to any product or service using any type of 802.11 technologies. It is the popular term for a high-frequency Wireless Local Area Network (WLAN). The Wi-Fi technology is rapidly gaining acceptance in many companies as an alternative to a wired LAN. Wi-Fi networks operate in the unlicensed 2.4 and $5 \mathrm{GHz}$ radio bands, with an $11 \mathrm{Mbps}$ (802.11b) or $54 \mathrm{Mbps}$ (802.11a) data rate, respectively. Along with the modern digital revolution, Wi-Fi like connectivity with increased speed and greater coverage area will gain increasing demand in coming years.

\section{Proposed Design}

\subsection{Scenario}

It is assumed that the communication of primary user is to working on a single frequency channel i.e., Figure 1 illustrated White Space. Now the main objective of the project is to detect white space in time domain. For designing as well as for implementation of communication network scenario and working on signal frequency channel i.e., white space in frequency domain is assumed. In this scenario, ten primary users and one secondary user are considered. The best way to facilitate equi-probable channel accessing is Time Division Multiple accessing

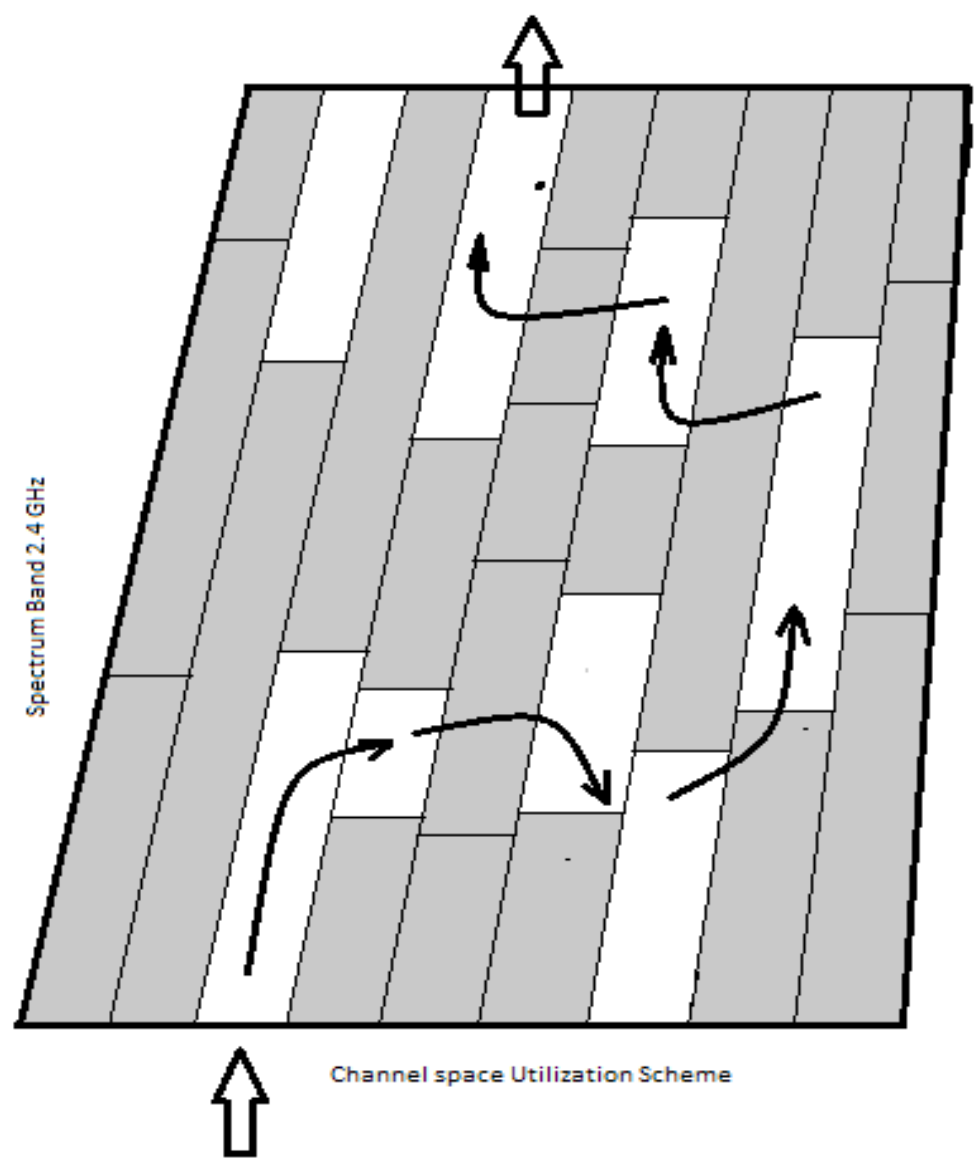

Figure 2. White space utilization by a secondary node on time domain mode. 
scheme. So, now the main task is to design a vacant space detection unit.

The white space detection module can be designed using microcontroller. The white space detection module detects the white space and shows its result on Serial Display and allots it to secondary node. The designs of these modules are discussed in the next sub-section. In order to observe the

Working of implemented design a UART module is additionally implemented to show output result on the PC. Figure 2 illustrated how the utilization of time domain white space by a secondary node is performed.

\subsection{Proposed Work}

The spectrum analyzer is used to display the $2.4 \mathrm{GHz}$ of ISM band in the range of 50 meter. In order to make an effective spectrum analyzer, this project used spectrum sensing device i.e. CYPRESS CYWM6935 (radio module). The Radio Module is a complete RF (Radio Frequency) transceiver module in the ISM band and is controlled by a microcontroller through Serial Peripheral Interface (SPI). The $2.4 \mathrm{GHz}$ transceiver chip contain RSSI (Receive Signal Strength Indicator) register that lets the chip continuously monitor the ISM band i.e., 2.4 to $2.483 \mathrm{GHz}$ how much signal power it is receiving. The detected signal will then display in the LCD. The LCD ensures that it will be able to display the detected signal strength. The various registers in the chip can be written to the microcontroller to set belongings like operating frequency as well as it can read other registers to recover data from the chip. The radio module is designed to function over the $2.4 \mathrm{GHz}$ band and has the capability to focus on a frequency band for finding any other devices that may be use the frequency in ISN band. The RF device is helping the microcontroller to select a suitably vacant space before transmitting the data.

The chip informs the signal level as a number normally up to 28 . A register value greater than 28 indicates that the received signal strength is strong, a Value lower than 10 indicates a low signal level with zero representing no signal. This facility can be used in this project i.e. sim- ply interface the microcontroller to instructs the module to go to that frequency then measure the signal strength of that frequency. When this is done, microcontroller steps the radio module to the next frequency and gives it the instruction to measure the signal level there. This process goes on right across the band. During this process is going on we will continuously monitor

- Signal to noise ration.

- Power of the signal.

Signal-to-noise ratio is also called as SNR or $\mathrm{S} / \mathrm{N}$, is defined as the ratio of signal power to the noise power corrupting the signal. The Signal to Noise Ratio (SNR) is the defining factor when it comes to quality of measurement. A high SNR guarantees clear acquisitions with low distortions and artefacts caused by noise. All real measurements are disturbed by noise. This includes electronic noise, but can also include external events that affect the measured phenomenon i.e. wind, vibrations, gravitational attraction of the moon, variations of temperature, variations of humidity, etc, depending on what is measured and of the sensitivity of the device. The concepts of signal-to-noise ratio and dynamic range are closely related. Dynamic range measures the ratio between the strongest un-distorted signal on a channel and the minimum discernible signal, which for most purposes is the noise level ${ }^{1}$. SNR measures the ratio between an arbitrary signal level (not necessarily the most powerful signal possible) and noise. Measuring signal-to-noise ratios requires the selection of a representative or reference signal ${ }^{4}$.

So for calculation Signal-to-noise ratio is defined as the ratio of the power of a signal (meaningful information) and the power of background noise (unwanted signal)

$$
\mathrm{SNR}=\mathrm{P}_{\text {signal }} / \mathrm{P}_{\text {noise }}
$$

Where $P$ is average power. Both signal and noise power must be measured at the same and equivalent points in a system, and within the same system bandwidth. If the 


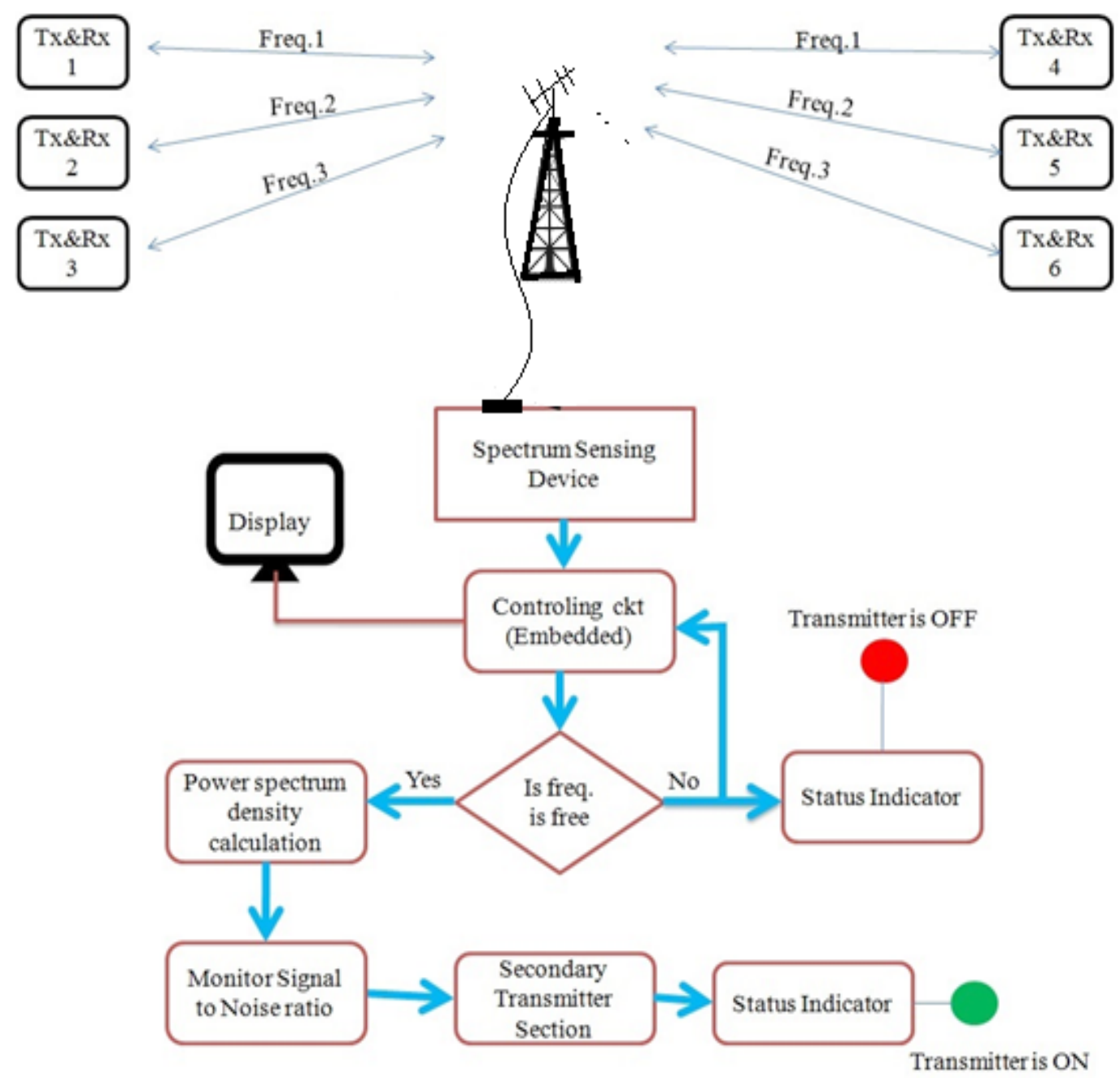

Figure 3. System flow for scanner module.

variance of the signal and noise are known, and the signal is zero-mean

$$
\mathrm{SNR}=\sigma_{\text {Signal }}^{2} / \sigma^{2}{ }_{\text {noise }}
$$

If the signal and the noise are measured across the same impedance, then the SNR can be obtained by calculating the square of the amplitude ratio

$$
\mathrm{SNR}=\mathrm{P}_{\text {Signal }} / \mathrm{P}_{\text {noise }}\left(\mathrm{A}_{\text {signal }} / \mathrm{A}_{\text {noise }}\right)
$$

Figure 3 illustrate the scanner ${ }^{3}$ is used to display the $2.4 \mathrm{GHz}$ of ISM band in the range of 50-100 meter. In order to make an effectual spectrum analyzer, in which a $2.4 \mathrm{GHz}$ transceiver chip contain RSSI-Receive Signal Strength Indicator register that lets the chip to monitor how much signal power it is receiving. From that register can saw the value of the power of that signal. Thus easily gates the exact value of the power from RSSI register. To gate the value once a connection has been established, the Channel register of radio module can be read to determine the relative connection quality of the channel' ${ }^{2}$. The 
register value indicates the strength of the received signal i.e., signal power. This is a read only value with the higher values indicating stronger received power of that channel. A Channel register value of $0-10$ indicates a channel that is relatively quiet that is the power of the signal is less. A Channel registers value greater than 10-28 indicates the channel is probably being used. A channel register value greater than 28 indicates the presence of a strong power of the signal 3.4 . To check for a lower power channel before transmitting, first set up receive mode properly and read the channel register. The detected signal will then display on the LCD 84x 48 pixels. Detailed steps wise algorithm is illustrated in Figure 4.

\section{Design Implementation Setup}

Step 1:As there are more chances of having authorized allocation of channels to other users, so in this

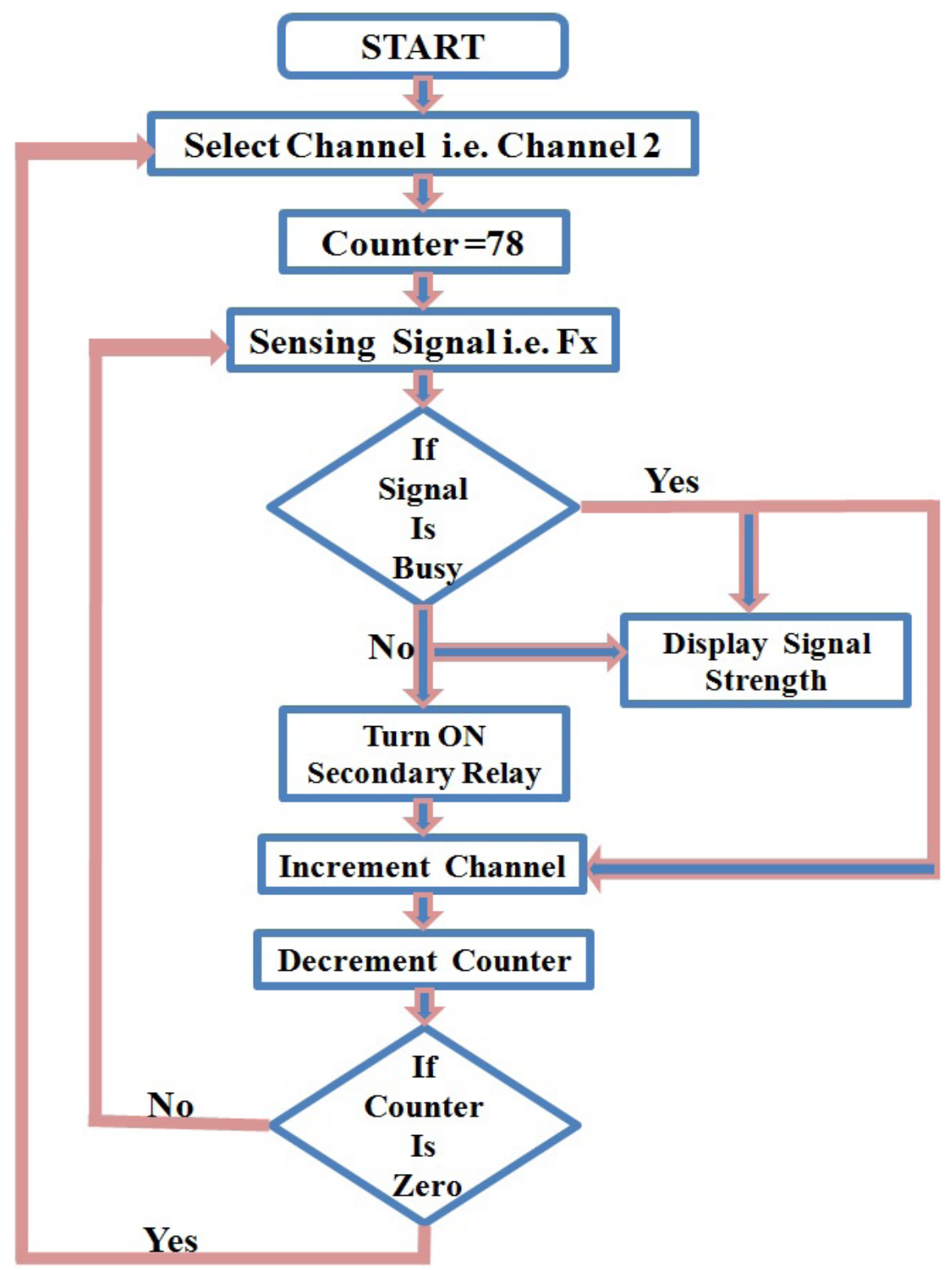

Figure 4. Design implementation for $2.4 \mathrm{GHz}$ scanner. 
work the channels would be selected taking into consideration the previous allocated channels. So channel from 2 onwards had been selected leaving channel 0 and 1 empty and any channel greater than 79 be avoided as by the rule of FCC because the selected channel does not interface with authorized user because the government provide the radio band i.e., $2.4 \mathrm{GHz}$ to $2.483 \mathrm{GHz}$.

Step 2:Device detecting the signal strength between the ranges from $2.402 \mathrm{GHz}$ to $2.482 \mathrm{GHz}$ to match the channel 2 with $2.402 \mathrm{GHz}$ we have to start the counter of no. 80 for sensing signal strength from channel 2 to 82 for does not sense the channel after $2.482 \mathrm{GHz}$.

Step 3:As the counter had been started, the first thing is to sense the selected channel frequency.

Step 4:While sensing, the user would be able to observe whether the channel is busy or not.

Step 5:If the selected channel is free, then secondary relay should indicate the respective frequency is vacant for further broadcasting. On the other hand if the selected channel is busy, then it should go to the next channel to sense the signal strength.

Step 6:Simultaneously the module should show the result on the display each time.

Step 7:As the next channel is selected, the number of counter should be decreased by one.

Step 8:Again counter will check the condition whether the count is equal to zero or not if not, then sensing of that particular channel continues otherwise the process for channel selection again resumes.

Step 9:Again the selection begins as the particular frequency is free for fraction of second. This process continues until the device is running on.

\section{Used Architecture}

The CYWM $69355^{14}$ Figure 5 illustrated detailed pin header with RFIN and RFOUT mode for Wireless USB Long

Table 1. Controller specification.

\begin{tabular}{|c|c|}
\hline Controller specification & ATmega328 \\
\hline Operating Voltage & $3.3 \mathrm{~V}$ \\
\hline Input Voltage (recommended) & $1.8-3.3 \mathrm{~V}$ \\
\hline Digital I/O Pins & 14 (of which 6 provide PWM output) \\
\hline Analog Input Pins & $40 \mathrm{~mA}$ \\
\hline DC Current per I/O Pin & $50 \mathrm{~mA}$ \\
\hline DC Current for 3.3V Pin & $2 \mathrm{~KB}$ \\
\hline Flash Memory & $32 \mathrm{~KB}$ of which $0.5 \mathrm{~KB} \mathrm{used} \mathrm{by} \mathrm{Boot} \mathrm{loader}$ \\
\hline SRAM & $16 \mathrm{MHz}$ \\
\hline Clock Speed & \\
\hline
\end{tabular}


Range (LR) Radio Module offers a complete radio module solution for integration into existing or new $2.4-\mathrm{GHz}$ products. The module is supplied with dual integrated PCB trace antennas. Which supported by $32 \mathrm{~KB}$ of flash memory, clock frequency $16 \mathrm{MHz}$ operates on 3.3 Voltage given in Table 1. The CYWM6935 is available in a small PCB design and can be mounted horizontally to the device PCB via a 12-pin header.

It can operate in the unlicensed ${ }^{5,6}$ ISM band $2.4 \mathrm{GHz}$ $2.483 \mathrm{GHz}$ and can be used in the range of 50 meter or more with the use of internal patch antenna ${ }^{12}$ as illus- trated in Figure 5(b). This complete radio module is selected because it has operating voltage of $2.7-3.6 \mathrm{~V}$ that is compatible with ATMEGA328 and LCD module. It also can be used in other application such as remote control, lighting control alarm and security.

The CYWM6935 is available in a small PCB design and can be mounted horizontally to the device PCB via a 12-pin header more details provided for special interfacing is illustrated as in Table 2 and 12 pin header pins is illustrated in Figure 6.

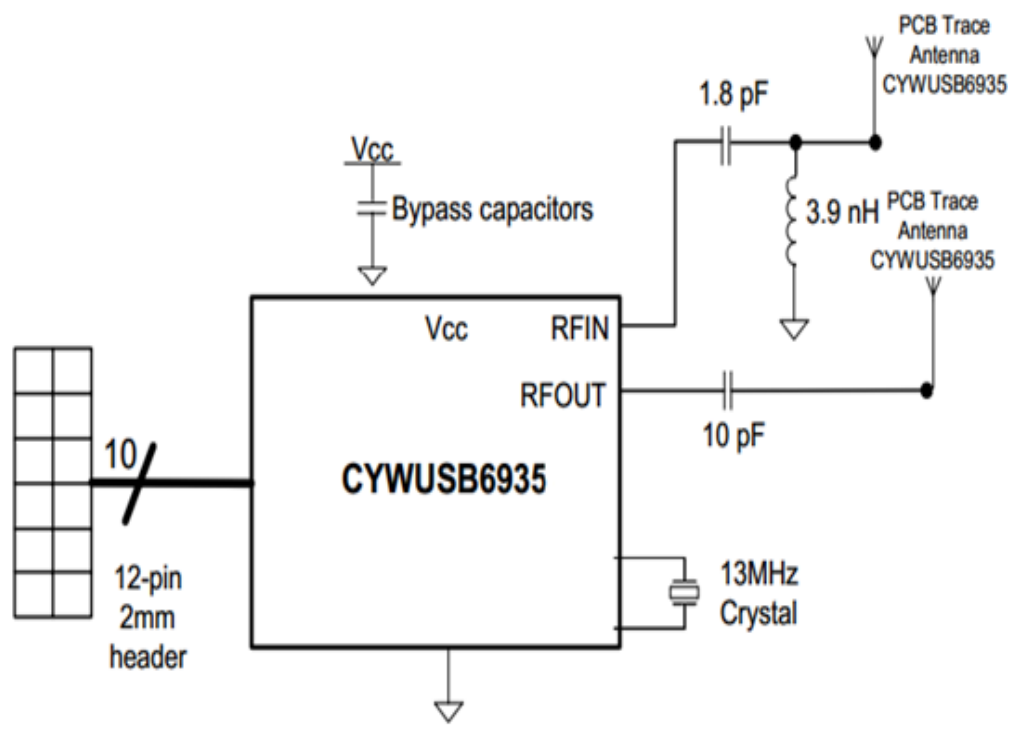

(a)

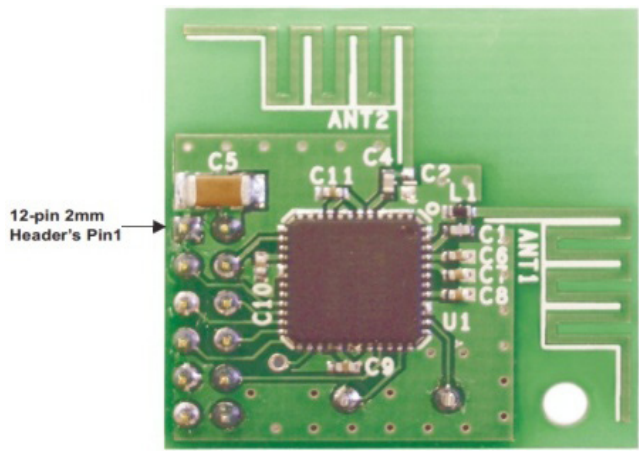

(b)

Figure 5. (a) $4 \mathrm{GHz}$ architecture with pin header. (b) A CYWM6935 with Internal Omni directional patch antenna. 
Table 2. CYWM6935 pins description

\begin{tabular}{|c|c|c|c|}
\hline Pin QFN & Name & Direction & Description \\
\hline 1 & GND & - & Ground \\
\hline 2 & VCC & - & $\begin{array}{l}\text { Supply voltage for the entire } \\
\text { radio module }(2.7 \mathrm{v}-3.6 \mathrm{v}) . \mathrm{It} \text { is } \\
\text { recommended that } 3.3 \mathrm{v} \text { be used for } \\
\text { the most applications }\end{array}$ \\
\hline 3 & IRQ & Output & $\begin{array}{c}\text { Interrupt signal from radio module } \\
\text { to the MCU. }\end{array}$ \\
\hline 4 & nRESET & Input & $\begin{array}{c}\text { Active low reset signal from MCU } \\
\text { to radio module }\end{array}$ \\
\hline 5 & MOSI & Input & $\begin{array}{l}\text { Master out, slave in SPI signal from } \\
\text { MCU to radio module }\end{array}$ \\
\hline 6 & nSS & Input & $\begin{array}{c}\text { Active low slave select signal from } \\
\text { MCU to radio module }\end{array}$ \\
\hline 7 & SCK & Input & $\begin{array}{l}\text { SPI clock from MCU to radio } \\
\text { module }\end{array}$ \\
\hline 8 & MISO & Output & $\begin{array}{l}\text { Master in, slave out SPI signal from } \\
\text { radio module to MCU ground }\end{array}$ \\
\hline 9 & GND & - & Ground \\
\hline 10 & $\mathrm{nPD}$ & Input & $\begin{array}{l}\text { Active low power-down signal from } \\
\text { MCU to radio module }\end{array}$ \\
\hline 11 & $\mathrm{~N} / \mathrm{C}$ & - & No connect-leave open \\
\hline 12 & $\mathrm{~N} / \mathrm{C}$ & - & No connect-leave open \\
\hline
\end{tabular}




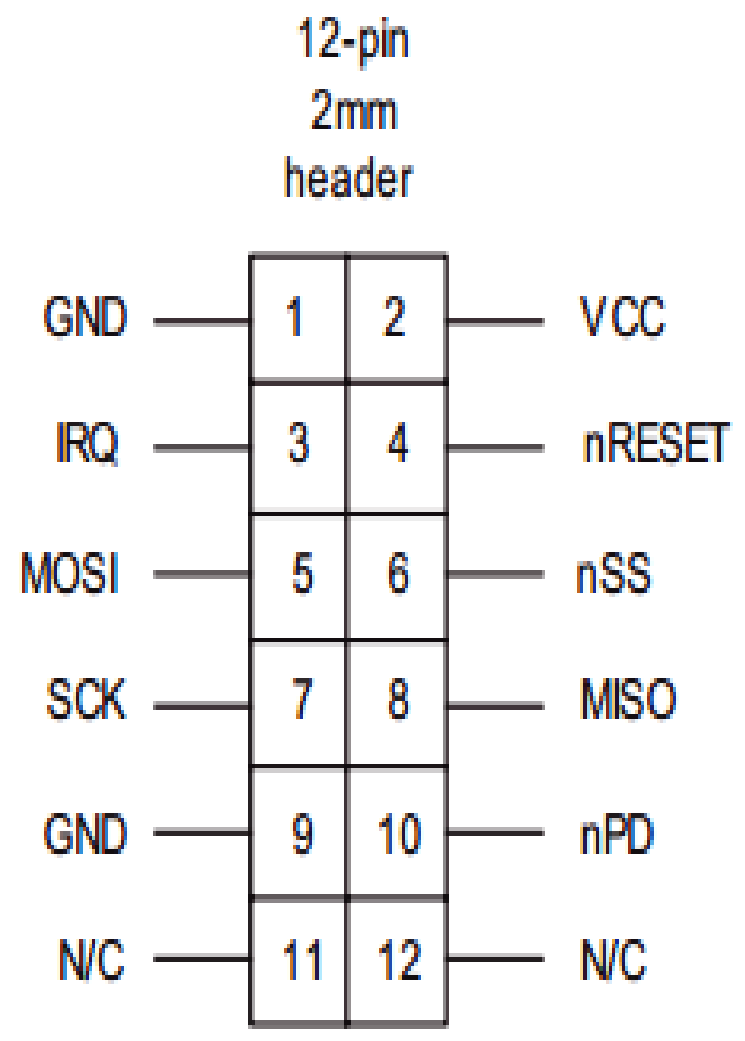

Figure 6. Header Pin out CYWM6935.

\section{Result and Testing}

Figure 7 illustrated a generic spectrum band scanner ${ }^{7}$.
The job and scope of the radio module is to conducts a test to verify device's ability to detect ISM signal. And it is control by the microcontroller using SPI interface.

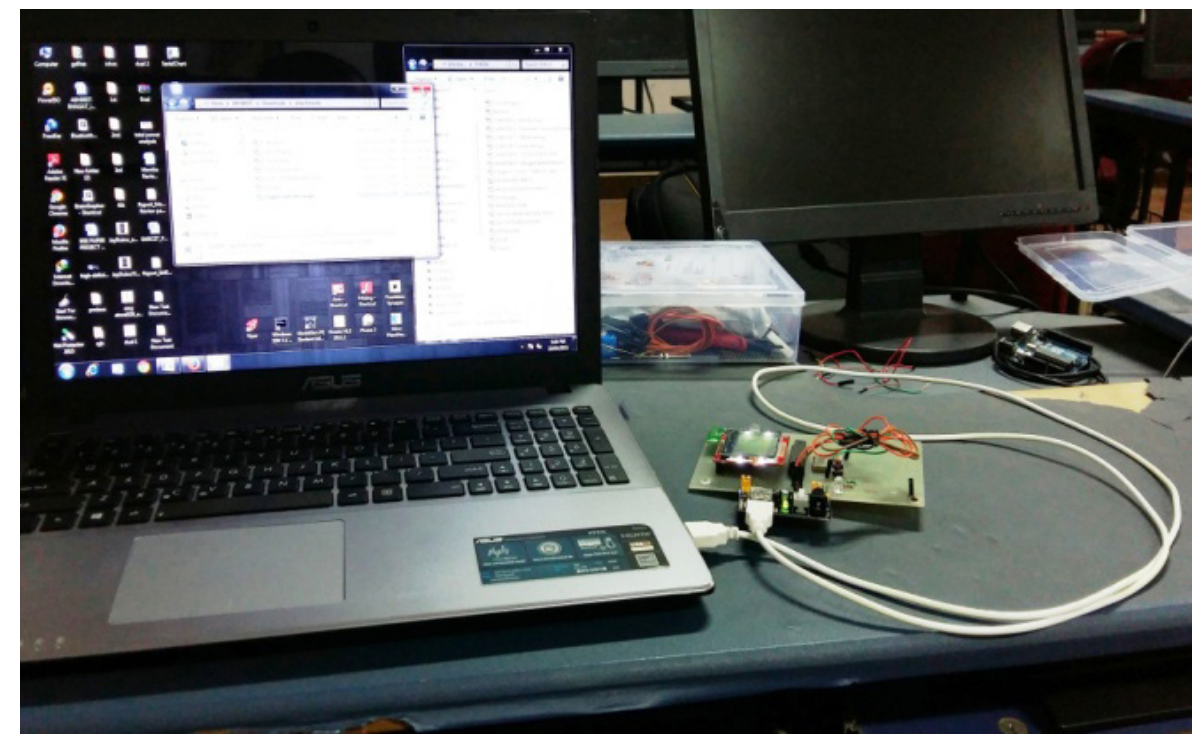

Figure 7. A generic spectrum band scanner CYWM6935. 


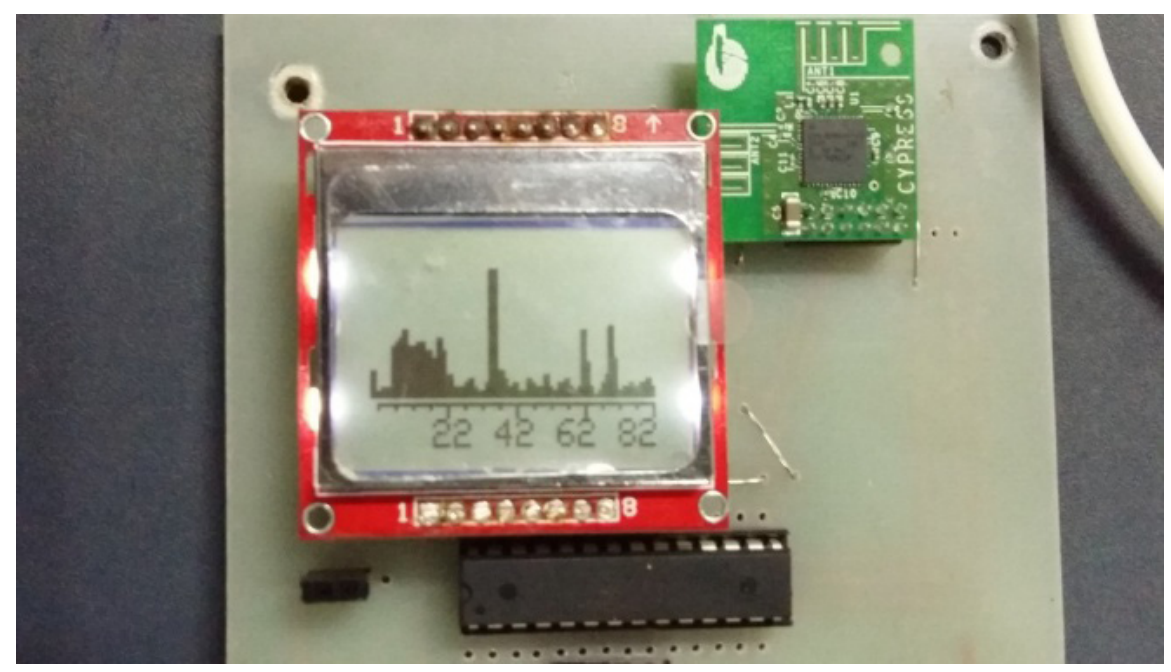

Figure 8. Test result at urban region.

The serial display is the graphical LCD of the Nokia 5110 phone. It feature is that it can run the serial data- ensures that it will be able to display the spectrum detected. Power source will implement the power system that has capability in providing 2.7 to $3.3 \mathrm{~V}$ and required current to each module in the whole board. The Spectrum sensing module from ISM band the real time data is to be sensing by the receiver module. The microcontroller is programmed such that radio module detects the signal level and that signal level is display on the serial display.
The results ${ }^{\underline{8}}$ of spectrum measurements had been presented in the ISM band prepared at three diverse places in the Nagpur area of India. One location is at the highly dense region in urban place. Another location is the semi urban area. A third location is in the outside of Nagpur, in a rural place. At each spectrum chart that shows variations in time and places, an economical spectrum sensing module has developed which used in White Space Networks.

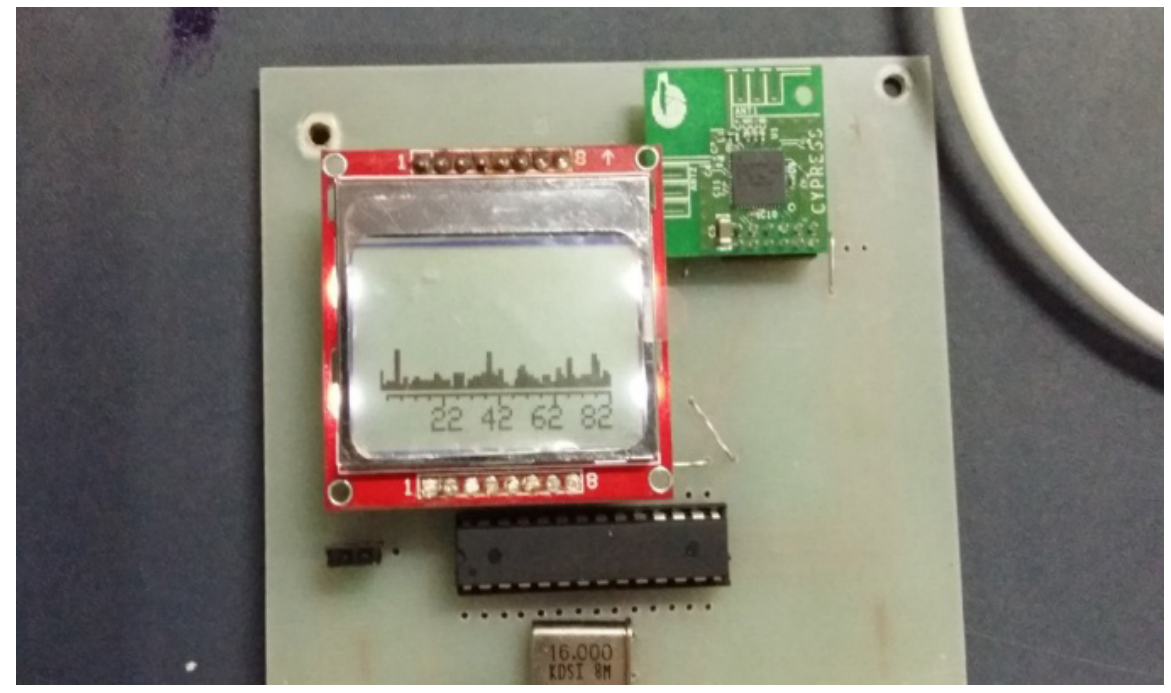

Figure 9. Test result at rural region. 


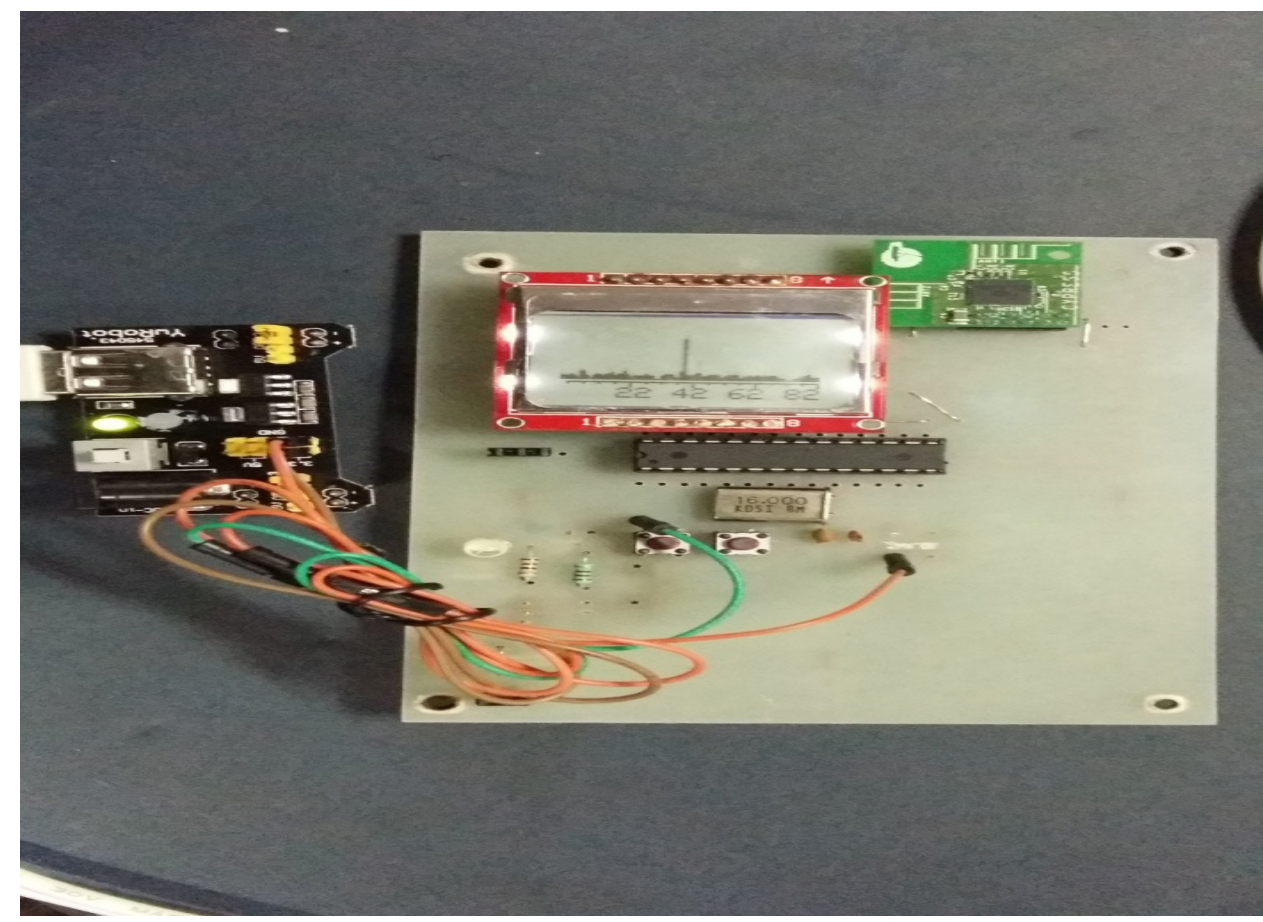

Figure 10. Test result-use of dedicated channel for duplex data transmission.

The signal 19 strengths at different frequencies has also been acquired throughout diverse times of the day at the different urban and rural areas. To analyze frequency and time spectrum utilization and usage the frequency usage, the graph is plot between frequency and signal strength $\frac{10}{}$. The spectrum sensing results at the region in urban location, Figure 8 shows that most of the bands which were observed for strong signals, had been used throughout the day. These were used for radio-location service. This $\underline{11}$ had been confirmed during repeated measurements in known vacant channels keep enough cushion for data transmission. Figure 9 shows the similar bands were observed in a rural region. The signal level become much attenuate and several bands vanish totally very quiet in the rural location. In rural location no signals were found in ISM band during that session. This experiment was for a rural region which is near to a large city. We analyzed that rural areas are located far away from the urban areas where spectrum occupancy is less and have larger vacant spaces than the earlier recorded areas.

Figure 10 shows that while searching the new Bluetooth user from one Bluetooth device, the user device scans the channels before sending the data to another device. Before transmitting the data the user device must be paired with the receiver device. The module that is designed in our system shows the channels which are used by transmitter device. Figure below shows the channels that are used by the transmitter device in searching the receiver device.

Scanning ${ }^{13}$ of the signals in rural area. At that time not even a single user was present who used the ISM band. When we transmitted the data of $5 \mathrm{~KB}$ from one mobile to another through Bluetooth communication, the signal 
strength is visible on the serial display. The figure shows the uses of proper use of channel while all other channels are vacant.

\section{Conclusion}

The ISM band is one of the most popular destinations for wireless standards for many reasons, one of which is the fact that it is a license-free band and opens to access any wireless device. Although it is a free-to-use kind of band, there are regulations and rules to be followed, and those rules are designed by the FCC to ensure fairness and innovation by the wireless devices. Peaceful coexistence between the wireless standards is important in the ISM band, and recently, an increasing concern has been given to this issue due to the fact that the numbers of users in the ISM band are increasing rapidly, which leads to many interference issues. A certainty is that characterizing the signals will help to overcome their interference effects, with the cognitive radio technique as the ultimate solution. Many models have been proposed for the cognitive radio technique.

In this thesis, a novel design has been proposed to utilize all these features to blindly identify the signals in order to evaluate how to overcome their effects. The decision making method will have a big impact on the spectrum awareness performance of the device, especially in a band like the ISM where every device can operate.

\section{Future Scope}

In future large number of devices will work on ISM band and the communication may be slow down in that case the module is present invention can provide the better way of communication by sending and receiving the data on vacant frequencies. This technology is used for fruitful, reliable and secured communication and monitoring in emergency and busy networks. There is a vast scope in future to the application of Vacant Spaces. Still now ISM band is very limited in which our present Wi-Fi systems are working. These wireless solutions are in great need for utilization of such white spaces to enhance their performance speed wise and coverage wise.

\section{References}

1. Silva MM, Ron CVR. Evaluation of white space detection algorithms for cognitive radio applications. SBMO/IEEE MTT-S International Microwave and Optoelectronics Conference (IMOC); 2013 Aug. p. 1-5. Crossref.

2. Kumarz P, Rakhejay N, Sarswaty A. White space detection and spectrum characterization in urban and rural India. IEEE 14th International Symposium and Workshops; 2013 Jun. p. 1-6.

3. Kim J, Jeffrey G. Sensitive white space detection with spectral covariance sensing. IEEE Transactions on Wireless Communications. 2010 Sep; 9(9):2945-55. Crossref.

4. Muraoka K, Sugahara H, Ariyoshi M. Monitoring-based spectrum management for expanding opportunities of white space utilization. IEEE International Symposium on Dynamic Spectrum Access Networks (DySPAN); 2011. p. 277-84. Crossref.

5. Naveen AAS, Zeng Y, Kumar VP. White space sensing device for detecting vacant channels in TV bands. 3rd International Conference; 2008 May. p. 1-6.

6. Subekti A, Sugihartono, Rachmana NS, Bayu AS. A blind spectrum sensing method for DTV signal detection. International Conference of Information and Communication Technology; 2013. p. 269-72.

7. Khan F, Nakagawa K. Comparative study of spectrum sensing techniques in cognitive radio networks. 2013 World Congress on Computer and Information Technology (WCCIT); 2013. p. 1-8. Crossref.

8. Bao N, Shen L. Quick sensing and spectrum sharing strategy for frequency hopping signal in ISM band. International Conference Wireless Communications and Signal processing, WCSP; 2009. p. 1-5. Crossref.

9. Biggs $\mathrm{M}$, Henley A, Clarkson T. Occupancy analysis of the $2.4 \mathrm{GHz}$ ISM band, IEE E Proceedings Communication. 2004 Oct; 151(5):481-8. Crossref.

10. Torabi N, Bhate S, Victor, Leung CM. Robust sensing strategy for dynamic spectrum access in the $2.4 \mathrm{GHz}$ ISM band. IEEE 24th International Symposium on Personal, Indoor 
and Mobile Radio Communications: Mobile and Wireless Networks; 2013. p. 2713-7.

11. Fulsunge AG, Nikhil P. Wyawahare of Electronics Engineering. Design and implementation for generating white space in a wi-fi like connectivity. International Journal of Engineering Research and Technology (IJERT). 2013 Mar; 2(3):1-8.

12. Tembhurne AP, Wyawahare NP. Alamouti based on spatial modulation for data transmission. International
Conference on Convergence of Technology; 2014. p. 1-4. Crossref.

13. Wyawahare NP, Haridas SL. Designing challenges to utilize white-space for configurable femtocell base on slotted TDM. IEEE, International Conference on Contemporary Computing and Informatics (IC3I); 2014. p. 1076-80. Crossref.

14. Wirelesssusb-lr-radio module. Available from: http://www. cypress.com/documentation/datasheets/cywm6935 